\title{
Notes on the Logic of Perfect Paradefinite Algebras
}

\author{
Joel Felipe Ferreira Gomes ${ }^{1}$, Vitor Rodrigues Greati ${ }^{1}$ \\ ${ }^{1}$ Programa de Pós-graduação em Sistemas e Computação (PPgSC) \\ Departamento de Informática e Matemática Aplicada \\ Universidade Federal do Rio Grande do Norte (UFRN) - Natal, RN - Brazil \\ \{joelffg,vitor.greati.017\}@ufrn.edu.br
}

\begin{abstract}
This work introduces the variety of perfect paradefinite algebras (PPalgebras), consisting of De Morgan algebras enriched with a perfect operator o, which turns out to be equivalent to the variety of involutive Stone algebras (IS-algebras). The corresponding order-preserving logic $\mathcal{P} \mathcal{P} \leq$ is a Logic of Formal Inconsistency, a Logic of Formal Undeterminedness, a $\boldsymbol{C}$-system and a $\boldsymbol{D}$-system, some of these features being evident in the proposed axiomatization of PP-algebras. After proving the mentioned algebraic equivalence, we show how to axiomatize, by means of Hilbert-style calculi, certain extensions of De Morgan algebras with a perfect operator and, in particular, the logic $\mathcal{P} \mathcal{P} \leq$.
\end{abstract}

\section{Introduction}

The variety of De Morgan algebras comprises all the bounded distributive lattices equipped with a De Morgan negation, that is, an involutive unary primitive operation satisfying the well-known De Morgan laws. Involutive Stone algebras (hereby called ISalgebras) are De Morgan algebras endowed with a primitive unary operation $\nabla$ that makes it possible to define a pseudo-complement $\neg$ satisfying the Stone identity $\neg x \vee \neg \neg x \approx \top$.

While the order-preserving logic canonically induced by De Morgan algebras, namely Belnap-Dunn's four-valued logic [Belnap 1977], has been extensively studied over the last decades, the logic so induced by IS-algebras, which we call $\mathcal{I S} \mathcal{S}_{\leq}$, has attracted attention only recently [Cantú 2019, Cantú and Figallo 2018]. Some of the most prominent features of such logic are the facts that it is paradefinite (paraconsistent and paracomplete, characteristics actually inherited from Belnap-Dunn logic), -gently explosive and $\sim$-gently implosive; in other words, it is a Logic of Formal Inconsistency (LFI) and a Logic of Formal Undeterminedness (LFU). More than that, $\mathcal{I} \mathcal{S}_{\leq}$belongs to the classes of $\mathbf{C}$-systems and $\mathbf{D}$-systems. (See [Marcos 2005a] for detailed explanations about all these concepts.)

All these philosophically rich characteristics are hidden in the presentation of ISalgebras in terms of $\nabla$, an operation whose interpretability and philosophical motivations are at best unclear. On the other hand, perfect operators (in the sense of [Marcos 2005b]) allow for the internalization of the very notion of consistency and determinedness at the object-language level, and have been extensively studied [Barrio and Carnielli 2019]. Given these observations, we propose, in this work, an alternative presentation of ISalgebras, which we will call perfect paradefinite algebras or simply PP-algebras, obtained by replacing $\nabla$ with a primitive perfect operation $\circ$. The proposed axiomatization

V. R. Greati acknowledges financial support from the Coordenação de Aperfeiçoamento de Pessoal de Nível Superior - Brasil (CAPES) - Finance Code 001. 
for these algebras will not only guarantee that the corresponding variety is term equivalent to the variety of IS-algebras, but it will also reflect the character of its induced order-preserving $\operatorname{logic} \mathcal{P} \mathcal{P} \leq$ as a $\mathbf{C}$-system and a $\mathbf{D}$-system. Furthermore, we will provide a Hilbert-style deduction system for logical matrices based on De Morgan algebras enriched with $\circ$, and, consequently, for the $\operatorname{logic} \mathcal{P} \mathcal{P} \leq$ itself.

\section{Algebraic and Logical Preliminaries}

A propositional signature is a family $\Sigma=\left\{\Sigma_{k}\right\}_{k \in \omega}$, where each $\Sigma_{k}$ is a collection of $k$-ary connectives. A $\Sigma$-algebra is a structure $\mathbf{A}=\left\langle A,{ }^{\cdot \mathbf{A}}\right\rangle$, where $A$ is a non-empty set called the carrier of $\mathbf{A}$ and, for each $\left(\mathcal{C} \in \Sigma_{k}\right.$, $C^{\mathbf{A}}: A^{k} \rightarrow A$ is the interpretation of (C) in A. Given a denumerable set $P \supseteq\{p, q, r, x, y\}$, the absolutely free algebra over $\Sigma$ freely generated by $P$, or simply the language over $\Sigma$ (generated by $P$ ), is denoted by $\mathbf{L}_{\Sigma}(P)$, and their elements are dubbed formulas, denoted here by Greek letters, like $\varphi, \psi$ and $\phi$. Given $\Sigma^{\prime} \subseteq \Sigma$ (that is, $\Sigma_{k}^{\prime} \subseteq \Sigma_{k}$ for all $k \in \omega$ ), the $\Sigma^{\prime}$-reduct of a $\Sigma$-algebra A is the $\Sigma^{\prime}$-algebra over the same carrier of $\mathbf{A}$ that agrees with $\mathbf{A}$ on the interpretation of the connectives in $\Sigma^{\prime}$. The collection of homomorphisms between two $\Sigma$-algebras A and B is denoted by $\operatorname{Hom}(\mathbf{A}, \mathbf{B})$, and the collection of mappings that are structure-preserving only in $\Sigma^{\prime} \subseteq \Sigma$ is denoted by $\operatorname{Hom}_{\Sigma^{\prime}}(\mathbf{A}, \mathbf{B})$. Furthermore, the set of endomorphisms on $\mathbf{A}$ is denoted by $\operatorname{End}(\mathbf{A})$ and each $\sigma \in \operatorname{End}\left(\mathbf{L}_{\Sigma}(P)\right)$ is called a substitution. Let $\varphi \in L_{\Sigma}(P)$ having $p_{1}, \ldots, p_{n}$ as propositional variables. We say that $\varphi$ is $n$-ary and denote by $\varphi^{\mathbf{A}}$ the $n$-ary operation on $A$ such that, for all $a_{1}, \ldots, a_{n} \in A, \varphi^{\mathbf{A}}\left(a_{1}, \ldots, a_{n}\right)=h(\varphi)$, for an $h \in$ $\operatorname{Hom}\left(\mathbf{L}_{\Sigma}(P), \mathbf{A}\right)$ with $h\left(p_{i}\right)=a_{i}$ for each $1 \leq i \leq n$. Also, if $\psi_{1}, \ldots, \psi_{n} \in L_{\Sigma}(P)$, we let $\varphi\left(\psi_{1}, \ldots, \psi_{n}\right)$ denote the formula $\varphi^{\mathbf{L}_{\Sigma}(P)}\left(\psi_{1}, \ldots, \psi_{n}\right)$. An equation is a pair $(\varphi, \psi)$ of formulas that we will denote $\varphi \approx \psi$, and a $\Sigma$-algebra A satisfies $\varphi \approx \psi$ iff $h(\varphi)=h(\psi)$ for every $h \in \operatorname{Hom}\left(\mathbf{L}_{\Sigma}(P), \mathbf{A}\right)$. A $\Sigma$-variety is a class of $\Sigma$-algebras that satisfies the same collection of equations. The variety generated by a class $\mathrm{K}$ of $\Sigma$-algebras, denoted by $\mathbb{V}(\mathrm{K})$, is the closure of $\mathrm{K}$ under homomorphic images, subalgebras and direct products.

We denote by $\Sigma^{\mathrm{L}}$ the signature containing but two binary connectives, $\wedge$ and $\vee$, and two nullary connectives $T$ and $\perp$, and by $\Sigma^{\mathrm{DM}}$ the extension of the latter with a unary connective $\sim$. Moreover, we let $\Sigma^{\mathrm{IS}}$ and $\Sigma^{\mathrm{PP}}$ be the signatures obtained from $\Sigma^{\mathrm{DM}}$ by adding unary connectives $\nabla$ and $\circ$, respectively. We provide below the definitions of De Morgan and involutive Stone algebras.

Definition 2.1. A De Morgan algebra is a $\Sigma^{\mathrm{DM}}$-algebra whose $\Sigma^{\mathrm{L}}$-reduct is a bounded distributive lattice, and satisfies the equations:

(DM1) $\sim \sim x \approx x \quad($ DM2 $) \sim(x \wedge y) \approx \sim x \vee \sim y$

Definition 2.2. An involutive Stone algebra (IS-algebra) is a $\Sigma^{\mathrm{IS}}$-algebra whose $\Sigma^{\mathrm{DM}}$ reduct is a De Morgan algebra, and satisfies the identities:

(IS1) $\nabla \perp \approx \perp($ IS2) $x \wedge \nabla x \approx x \quad($ IS3) $\nabla(x \wedge y) \approx \nabla x \wedge \nabla y$ (IS4) $\sim \nabla x \wedge \nabla x \approx \perp$

In this work, we denote by $\mathbb{I S}$ the variety of IS-algebras. The next result presents some equations satisfied by IS-algebras, which will be useful for proving the results of the next section. 
Lemma 2.3. The following equations are satisfied by IS-algebras:
1. $x \vee \nabla \sim x \approx \top$
2. $\sim \nabla(x \wedge \sim x) \wedge \sim x \approx \sim \nabla x$
3. $x \wedge \sim \nabla x \approx \perp$
4. $\nabla \nabla x \approx \nabla x$
5. $\nabla \sim \nabla x \approx \sim \nabla x$
6. $\sim \nabla \sim(x \wedge y) \approx \sim \nabla \sim x \wedge \sim \nabla \sim y$

A (logical) $\Sigma$-matrix $\mathfrak{M}$ is a structure $\langle\mathbf{A}, D\rangle$ such that $\mathbf{A}$ is a $\Sigma$-algebra and $D \subseteq A$. The mappings in $\operatorname{Hom}\left(\mathbf{L}_{\Sigma}(P), \mathbf{A}\right)$ are dubbed $\mathfrak{M}$-valuations. In this work, a SET-FMLA logic is a consequence relation $\vdash \subseteq \wp L_{\Sigma}(P) \times L_{\Sigma}(P)$ and a SET-SET logic is a generalized consequence relation $\triangleright \subseteq \wp L_{\Sigma}(P) \times \wp L_{\Sigma}(P)$. The complement of a given SET-SET logic $\triangleright$ will be denoted by $\downarrow$. Every $\Sigma$-matrix induces a SETSET logic $\triangleright_{\mathfrak{M}}$ such that $\Gamma \triangleright \mathfrak{M} \Delta$ iff, for all $h \in \operatorname{Hom}\left(\mathbf{L}_{\Sigma}(P), \mathbf{A}\right), h(\Gamma) \cap \bar{D} \neq \varnothing$ or $h(\Delta) \cap D \neq \varnothing$ as well as a SET-FMLA logic $\vdash_{\mathfrak{M}}$ with $\Gamma \vdash_{\mathfrak{M}} \varphi$ iff $\Gamma \triangleright_{\mathfrak{M}}\{\varphi\}$. Whenever $\triangleright_{\mathfrak{M}}=\triangleright$ (resp. $\left.\vdash_{\mathfrak{M}}=\vdash\right)$, we say that $\mathfrak{M}$ characterizes $\triangleright$ (resp. $\vdash$ ). Based on [Shoesmith and Smiley 1978, Caleiro and Marcelino 2019], we define a symmetrical (Hilbert-style) calculus $\mathrm{R}$ as a collection of pairs $\frac{\Gamma}{\Delta}=(\Gamma, \Delta) \in \wp L_{\Sigma}(P) \times \wp L_{\Sigma}(P)$, called (symmetrical) rules of inference, where $\Gamma$ is the antecedent and $\Delta$ is the succedent of the rule. We then say that $\Gamma \triangleright_{\mathrm{R}} \varphi$ whenever there is a rooted tree whose root is labelled with $\Gamma$, every non-leaf node results from an expansion of its parent by means of a substitution instance of an inference rule of $R$ and each leaf is either discontinued by effect of an inference rule with empty succedent or intersects $\Delta$. The relation $\triangleright_{\mathrm{R}}$ so defined is a SET-SET logic and, when $\triangleright_{\mathrm{R}}=\triangleright_{\mathfrak{M}}$, we say that $\mathrm{R}$ axiomatizes $\mathfrak{M}$. A rule $\frac{\Gamma}{\Delta}$ is sound in $\mathfrak{M}$ when $\Gamma \triangleright_{\mathfrak{M}} \Delta$. It is easy to notice that this deductive formalism generalizes Hilbert calculi in the usual sense, these being those symmetrical calculi whose rules have finite antecedents and only singletons as succedents. Finally, every $\Sigma$-variety K such that each $\mathrm{A} \in \mathrm{K}$ has a meet-semilattice reduct with top element $T$ induces a SET-FMLA orderpreserving logic $\vdash_{\mathrm{K}}^{<}$according to which $\varphi$ follows from $\Gamma$ iff $\Gamma=\varnothing$ and $T \approx \varphi$ or there are $\gamma_{1}, \ldots, \gamma_{n} \subseteq \Gamma(n \geq 1)$ such that the identity $\bigwedge_{i} \gamma_{i} \approx \bigwedge_{i} \gamma_{i} \wedge \varphi$ is valid in $\mathrm{K}$.

\section{Perfect Paradefinite Algebras}

We propose in this section to extend De Morgan algebras with a perfect operator $\circ$. In the sequel, we will prove that the variety of such algebras is equivalent to the variety of IS-algebras.

Definition 3.1. A perfect paradefinite algebra (PP-algebra) is a $\Sigma^{\mathrm{PP}}$-algebra whose $\Sigma^{\mathrm{DM}}$ reduct is a De Morgan algebra, and satisfies the following equations: (PP1) $\circ \circ x \approx \top \quad($ PP2 $) \circ x \approx \circ \sim x \quad$ (PP3) $\circ \top \approx \top \quad($ PP4 $) \circ x \wedge(\sim x \wedge x) \approx \perp$
$($ PP5 $\circ(x \wedge y) \approx(\circ x \vee \circ y) \wedge(\circ x \vee \sim y) \wedge(\circ y \vee \sim x)$

We denote by $\mathbb{P P}$ the variety of PP-algebras. The following result presents some useful equations and inequalities satisfied by the members of $\mathbb{P P}$.

Lemma 3.2. Every PP-algebra satisfies:

$$
\begin{aligned}
& \text { 1. } \sim \circ x \vee(x \vee \sim x) \approx \top \quad \text { 2. } \circ x \wedge \sim \circ x \approx \perp \quad \text { 3. } \circ x \leq x \vee \sim x \\
& \text { If } \varphi \in L_{\Sigma^{\mid S}}(P)\left(\text { resp. } \varphi \in L_{\Sigma^{\mathrm{PP}}}(P)\right) \text {, let } \varphi^{\circ} \in L_{\Sigma^{\mathrm{PP}}}(P)\left(\operatorname{resp} . \varphi^{\nabla} \in L_{\Sigma^{\mathrm{IS}}}(P)\right)
\end{aligned}
$$

be the result of applying the definition of $\circ$ (resp. of $\nabla$ ) given below, in Theorem 3.3 (Theorem 3.4), over $\varphi$. Extend this notion to sets of formulas in the usual way. The following results show that the varieties of involutive Stone algebras and perfect paradefinite algebras are equivalent. 
Theorem 3.3. Let $\mathbf{A} \in \mathbb{I S}$. Then the $\Sigma^{\mathrm{PP}}$-algebra $\mathbf{A}^{\circ}$, with $\circ x=\sim \nabla(x \wedge \sim x)$, is $a$ PP-algebra.

Proof. We will show that $\mathbf{A}^{\circ}$ satisfies all the characteristic equations of PP-algebras.

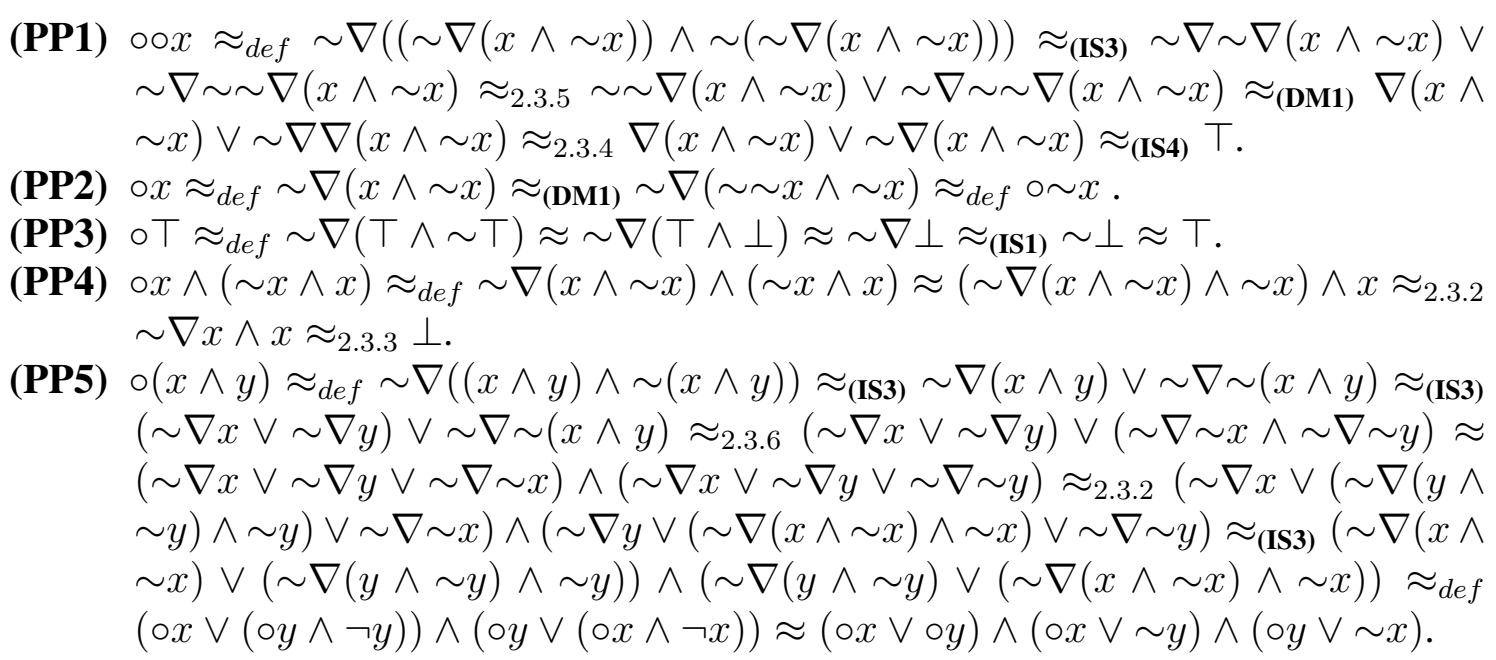

Theorem 3.4. Let $\mathbf{A} \in \mathbb{P P}$. Then the $\Sigma^{\mathrm{IS}}$-algebra $\mathbf{A}^{\nabla}$, with $\nabla x=\sim \circ x \vee x$, is an IS-algebra.

Proof. We will show that $\mathbf{A}^{\nabla}$ satisfies all the characteristic equations of IS-algebras.

(IS1) $\nabla \perp \approx_{\text {def }} \sim 0 \perp \vee \perp \approx \sim 0 \perp \approx \sim 0 \sim \top \approx_{(\mathbf{P P 2})} \sim 0 \top \approx_{(\mathbf{P P 3})} \sim \top \approx \perp$.

(IS2) By absorption and commutativity of $\vee$, we have $x \wedge \nabla x \approx_{\text {def }} x \wedge(\sim \circ x \vee x) \approx x$.

(IS3) $\nabla(x \wedge y) \approx_{d e f} \sim \circ(x \wedge y) \vee(x \wedge y) \approx_{(\mathbf{P P 5})}(\sim \circ x \wedge \sim \circ y) \vee(\sim \circ x \wedge y) \vee(\sim \circ$ $y \wedge x) \vee(x \wedge y) \approx(\sim \circ x \vee x) \wedge(\sim \circ y \vee y) \approx_{\text {def }} \nabla x \wedge \nabla y$.

(IS4) $\sim \nabla x \wedge \nabla x \approx_{d e f} \sim(\sim \circ x \vee x) \wedge(\sim \circ x \vee x) \approx_{(\text {DM2 })}(\circ x \wedge \sim x) \wedge(\sim \circ x \vee x) \approx$ $(\circ x \wedge \sim x \wedge \sim 0 x) \vee(\circ x \wedge \sim x \wedge x) \approx_{(\mathbf{P P 4})}(\circ x \wedge \sim x \wedge \sim \circ x) \vee \perp \approx 0 x \wedge \sim x \wedge \sim 0 x \approx$ $\circ x \wedge \sim x \wedge \sim \circ x \wedge \top \approx_{(\mathbf{P P 1})} \circ x \wedge \sim x \wedge \sim \circ x \wedge \circ \circ x \approx_{(\mathbf{P P} 4)} \perp \wedge \sim x \approx \perp$.

Theorem 3.5. Given $\mathbf{A} \in \mathbb{I S}$ and $\mathbf{B} \in \mathbb{P P}$, we have $\left(\mathbf{A}^{\circ}\right)^{\nabla}=\mathbf{A}$ and $\left(\mathbf{B}^{\nabla}\right)^{\circ}=\mathbf{B}$.

Proof. In order to prove that $\left(\mathbf{A}^{\circ}\right)^{\nabla}=\mathbf{A}$, it is enough to show that $\sim(\sim \nabla(x \wedge \sim x)) \vee x \approx$ $\nabla x$ holds in $\mathbf{A}$, that is, the operation induced by the term $\left((\nabla x)^{\circ}\right)^{\nabla}$ is the same as $\nabla$. By the fact that $\nabla x \vee x \approx \nabla x$, we have $\sim(\sim \nabla(x \wedge \sim x)) \vee x \approx_{(\text {DM1 })} \nabla(x \wedge \sim x) \vee x \approx_{(\text {IS3) }}$ $(\nabla x \wedge \nabla \sim x) \vee x \approx(\nabla x \vee x) \wedge(\nabla \sim x \vee x) \approx_{2.3 .1}(\nabla x \vee x) \wedge \top \approx \nabla x \vee x \approx \nabla x$. Similarly, for proving $\left(\mathbf{B}^{\nabla}\right)^{\circ}=\mathbf{B}$, it is enough to show that $\left((\circ x)^{\nabla}\right)^{\circ}$ induces an operation equal to $\circ$, which amounts to proving that $\sim(\sim \circ(x \wedge \sim x) \vee(x \wedge \sim x)) \approx \circ x$ holds in $\mathbf{B}$. Then, we have $\sim(\sim \circ(x \wedge \sim x) \vee(x \wedge \sim x)) \approx_{(\text {DM2 })} \circ(x \wedge \sim x) \wedge(\sim x \vee x) \approx_{(\text {PP5) }}(\circ x \vee \circ \sim x) \wedge$ $(\circ x \vee x) \wedge(\mathrm{\circ} \sim x \vee \sim x) \wedge(\sim x \vee x) \approx_{(\mathbf{P P 2})}(\circ x \vee \circ x) \wedge(\circ x \vee x) \wedge(\circ x \vee \sim x) \wedge(\sim x \vee x) \approx$ $\circ x \wedge(\sim x \vee x) \approx_{3.2 .3} \circ x$.

We denote by $\mathbf{I S}_{6}$ the subdirectly irreducible six-element ISalgebra [Cantú and Figallo 2018] and by $\mathbf{P P}_{6}$ the algebra $\mathbf{I S}_{\mathbf{6}}{ }^{\circ}$ (defined according to Theorem 3.3). As a corollary of the equivalence just presented and of a similar result for IS-algebras [Marcelino and Rivieccio 2021], we have that the variety of PP-algebras is generated by $\mathrm{PP}_{6}$. Let $\mathcal{P} \mathcal{P} \leq$ be the order-preserving logic induced by $\mathbb{P P}$. We will use the following technical results to prove that this logic is characterized by a single logical matrix. 
Lemma 3.6. Given $\mathbf{A} \in \mathbb{I S}$ and $\mathbf{B} \in \mathbb{P P}$,

1. if $h \in \operatorname{Hom}\left(\mathbf{L}_{\Sigma^{\mathrm{IS}}}(P), \mathbf{A}\right)$, then $h\left(\left(\varphi^{\circ}\right)^{\nabla}\right)=h(\varphi)$ for all $\varphi \in L_{\Sigma^{\mathrm{IS}}}(P)$;

2. if $h \in \operatorname{Hom}\left(\mathbf{L}_{\Sigma^{\mathrm{PP}}}(P), \mathbf{B}\right)$, then $h\left(\left(\varphi^{\nabla}\right)^{\circ}\right)=h(\varphi)$ for all $\varphi \in L_{\Sigma^{\mathrm{PP}}}(P)$;

3. if $h \in \operatorname{Hom}\left(\mathbf{L}_{\Sigma^{1 S}}(P), \mathbf{A}\right)$, then the mapping $h^{\circ} \in \operatorname{Hom}\left(\mathbf{L}_{\Sigma^{\mathrm{PP}}}(P), \mathbf{A}^{\circ}\right)$ such that $h^{\circ}(p)=h(p)$ for all $p \in P$ satisfies $h^{\circ}\left(\varphi^{\circ}\right)=h(\varphi)$ for all $\varphi \in L_{\Sigma^{1 S}}(P)$;

4. if $h \in \operatorname{Hom}\left(\mathbf{L}_{\Sigma^{P P}}(P), \mathbf{B}\right)$, then the mapping $h^{\nabla} \in \operatorname{Hom}\left(\mathbf{L}_{\Sigma^{1 S}}(P), \mathbf{B}^{\nabla}\right)$ such that $h^{\nabla}(p)=h(p)$ for all $p \in P$ satisfies $h^{\nabla}\left(\varphi^{\nabla}\right)=h(\varphi)$ for all $\varphi \in L_{\Sigma^{\mathrm{PP}}}(P)$.

Proof. By induction on the structure of formulas and Theorem 3.5.

Proposition 3.7. In what follows, let $\mathbf{A} \in \mathbb{P P}$. Then,

1. $\Gamma \vdash_{\langle\mathbf{A}, D\rangle} \varphi$ iff $\Gamma^{\nabla} \vdash_{\left\langle\mathbf{A}^{\nabla}, D\right\rangle} \varphi^{\nabla}$

2. $\Gamma \vdash_{\mathcal{P P}} \varphi$ iff $\Gamma^{\nabla} \vdash_{\mathcal{I} \mathcal{S}_{\leq}} \varphi^{\nabla}$

Proof. Follows by Lemma 3.6.

Theorem 3.8. $\mathcal{P} \mathcal{P}_{\leq}=\vdash_{\left\langle\mathbf{P P}_{6}, \uparrow a\right\rangle}$.

Proof. By Proposition 3.7 and the fact that $\vdash_{\mathcal{I} \mathcal{S}_{<}}$is characterized by the matrix $\left\langle\mathbf{I S}_{\mathbf{6}}, \uparrow a\right\rangle$, we have $\Gamma \vdash_{\left\langle\mathbf{P P}_{\mathbf{6}}, \uparrow a\right\rangle} \varphi$ iff $\Gamma^{\nabla} \vdash_{\left\langle\mathbf{I S}_{6}, \uparrow a\right\rangle} \varphi^{\nabla}$ iff $\Gamma^{\nabla} \vdash_{\mathbf{I S}_{\mathbf{6}}} \varphi^{\nabla}$ iff $\Gamma \vdash_{\mathcal{P P}} \varphi$.

Finally, following [Marcelino and Rivieccio 2021], we present a recipe to produce a PP-algebra by extending a given De Morgan algebra. We will see then how to axiomatize logics induced by the former having a calculus for logics induced by the latter.

Definition 3.9. Let $\mathbf{A}$ be a $\Sigma^{\mathrm{DM}}$-algebra. Given $\hat{0}, \hat{1} \notin A$, we define the $\Sigma^{\mathrm{PP}}$-algebra $\mathbf{A}^{\circ}=\left\langle A \cup\{\hat{0}, \hat{1}\}, \cdot^{\circ}\right\rangle$ by letting

$$
\begin{aligned}
& { }^{\circ} \mathbf{A}^{\circ} a=\left\{\begin{array}{ll}
\hat{1} & \text { if } a=\hat{0} \text { or } a=\hat{1} \\
\hat{0} & \text { otherwise }
\end{array} \quad a \wedge \mathbf{A}^{\circ} b= \begin{cases}a \wedge^{\mathbf{A}} b & \text { if } a, b \in A \\
\hat{1} & \text { if } a=b=\hat{1} \\
\hat{0} & \text { if } a=\hat{0} \text { or } b=\hat{0} \\
c & \text { if }\{a, b\}=\{\hat{1}, c\} \text { with } c \in A\end{cases} \right. \\
& \sim^{\mathbf{A}^{\circ}} a=\left\{\begin{array}{ll}
\sim^{\mathbf{A}} a & \text { if } a \in A \\
\hat{0} & \text { if } a=\hat{1} \\
\hat{1} & \text { if } a=\hat{0}
\end{array} \quad a \vee^{\mathbf{A}^{\circ}} b= \begin{cases}a \vee^{\mathbf{A}} b & \text { if } a, b \in A \\
\hat{0} & \text { if } a=b=\hat{0} \\
\hat{1} & \text { if } a=\hat{1} \text { or } b=\hat{1} \\
c & \text { if }\{a, b\}=\{\hat{0}, c\} \text { with } c \in A\end{cases} \right. \\
& \perp^{\mathbf{A}^{\circ}}=\hat{0} \quad \top^{\mathbf{A}^{\circ}}=\hat{1}
\end{aligned}
$$

Proposition 3.10. Whenever $\mathbf{A}$ is a De Morgan algebra, $\mathbf{A}^{\circ}$ is a PP-algebra.

\section{Axiomatizing Logics of De Morgan Algebras Enriched with a Perfect Operator}

Given a $\Sigma^{\mathrm{DM}}$-matrix $\mathfrak{M}=\langle\mathbf{A}, D\rangle$, let $\mathfrak{M}^{\circ}=\left\langle\mathbf{A}^{\circ}, D \cup\{\hat{1}\}\right\rangle$ be the $\Sigma^{\mathrm{PP}}$-matrix with the underlying (by Proposition 3.10, perfect paradefinite) algebra $\mathrm{A}^{\circ}$ given by Definition 3.9. We denote by $\widehat{\mathfrak{M}}$ the $\Sigma^{\mathrm{DM}}$-reduct of $\mathfrak{M}^{\circ}$. Given a class of $\Sigma^{\mathrm{DM}}$-matrices $\mathcal{M}$, we let $\mathcal{M}^{\circ}:=$ $\left\{\mathfrak{M}^{\circ}: \mathfrak{M} \in \mathcal{M}\right\}$ and $\widehat{\mathcal{M}}:=\{\widehat{\mathfrak{M}}: \mathfrak{M} \in \mathcal{M}\}$. In what follows, if $\triangleright_{1}$ and $\triangleright_{2}$ are SETSET logics over $\Sigma$, we let $\triangleright_{1} \simeq \triangleright_{2}$ iff $\triangleright_{1} \cup\left\{\left(L_{\Sigma}(P), \varnothing\right)\right\}=\triangleright_{2} \cup\left\{\left(L_{\Sigma}(P), \varnothing\right)\right\}$. The 
following result gives a generic recipe for axiomatizing the SET-SET logic determined by the class $\mathcal{M}^{\circ}$, assuming we have a calculus $\mathrm{R}$ that axiomatizes the SET-SET logic determined by $\widehat{\mathcal{M}}$.

Theorem 4.1. Let $\mathcal{M}$ be a class of $\Sigma^{\mathrm{DM}}$-matrices. If $\triangleright_{\widehat{\mathcal{M}}} \simeq \triangleright_{\mathrm{R}}$, then $\triangleright_{\mathcal{M}^{\circ}}=\triangleright_{\mathrm{R} \cup \mathrm{R}_{\circ}}$, where $\mathrm{R}_{\circ}$ consists of the following rules of inference:

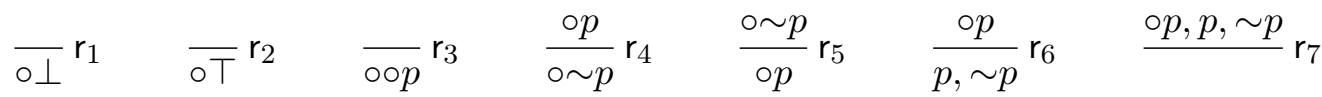

$$
\begin{aligned}
& \frac{\circ p}{\circ(p \wedge q), p} \mathrm{r}_{8} \quad \frac{\circ q}{\circ(p \wedge q), q} \mathrm{r}_{9} \quad \frac{\circ(p \wedge q), q}{\circ p} \mathrm{r}_{10} \quad \frac{\circ(p \wedge q), p}{\circ q} \mathrm{r}_{11} \\
& \frac{\circ p, \circ q}{\circ(p \wedge q)} r_{12} \quad \frac{\circ(p \wedge q)}{\circ p, \circ q} r_{13} \quad \frac{\circ p, \circ q}{\circ(p \vee q)} r_{14} \quad \frac{\circ(p \vee q)}{\circ p, \circ q} r_{15} \\
& \frac{\circ p, p}{\circ(p \vee q)} r_{16} \quad \frac{\circ q, q}{\circ(p \vee q)} r_{17} \quad \frac{\circ(p \vee q)}{\circ p, q} r_{18} \quad \frac{\circ(p \vee q)}{\circ q, p} r_{19}
\end{aligned}
$$

Proof. Checking the soundness of those rules is routine; we give only a couple of examples. Let $v$ be a valuation over a matrix $\mathfrak{M}^{\circ}$. The rule $\mathrm{r}_{3}$ is sound in $\mathfrak{M}^{\circ}$, given that, if $v(\circ \varphi) \in\{\hat{0}, \hat{1}\}$, then we have that $v(\circ \circ \varphi)=\hat{1}$. Regarding rule $r_{4}$, we have that, if $v(\circ \varphi)=\hat{1}$, then $v(\varphi) \in\{\hat{0}, \hat{1}\}$. So $v(\sim \varphi) \in\{\hat{0}, \hat{1}\}$, thus $v(\circ \sim \varphi)=\hat{1}$. For completeness, assume $\Gamma \nabla_{\mathrm{R}_{0}} \Delta$. Then, by cut of sets, there is a partition $\langle T, F\rangle$ of $L_{\Sigma^{\mathrm{PP}}}(P)$ such that $\Gamma \subseteq T$ and $\Delta \subseteq F$ and $T \mathrm{R}_{\circ} F$. Note that (by $r_{3}, r_{6}$ and $r_{7}$ ) for each $\varphi$, we have either $\circ \varphi \in T$ or $\sim \circ \varphi \in T$, but never both. In particular, $F$ is never empty. Also, by $r_{6}$ and $r_{7}$, if we have $\circ \varphi \in T$, we have either $\varphi \in T$ or $\sim \varphi \in T$, but never both. Hence, each $\varphi$ must be exactly in one of three cases: (a) $\sim \circ \varphi \in T$, (b) $\circ \varphi, \varphi \in T$ or (c) $\circ \varphi, \sim \varphi \in T$. Since $\mathrm{R} \subseteq \mathrm{R} \cup \mathrm{R}_{\circ}$, we also have $T \nabla_{\mathrm{R}} F$. From the fact that $\triangleright_{\mathrm{R}} \simeq \triangleright_{\widehat{\mathfrak{M}}}$ and $F \neq \emptyset$ we know that $T \widehat{\mathfrak{M}}_{\widehat{M}} F$. We can therefore pick $v \in \operatorname{Hom}_{\Sigma^{\mathrm{DM}}}\left(\mathbf{L}_{\Sigma^{\mathrm{PP}}}(P), \widehat{\mathfrak{M}}\right)$, for some $\mathfrak{M} \in \mathcal{M}$, such that $v(T) \subseteq D$ and $v(F) \cap D=\emptyset$. Consider $v^{\prime}: L_{\Sigma^{\mathrm{PP}}}(P) \rightarrow \mathfrak{M}^{\circ}$ defined by:

$$
v^{\prime}(\varphi):= \begin{cases}v\left(\varphi_{i}\right) & \text { if } \varphi=\varphi_{1} \wedge \varphi_{2} \text { and } \sim \circ \varphi, \circ \varphi_{3-i} \in T \\ v\left(\varphi_{i}\right) & \text { if } \varphi=\varphi_{1} \vee \varphi_{2} \text { and } \sim \circ \varphi, \circ \varphi_{i} \in T \\ v(\varphi) & \text { if } \sim \circ \varphi \in T \\ \hat{1} & \text { if } \circ \varphi, \varphi \in T \\ \hat{0} & \text { if } \circ \varphi, \sim \varphi \in T\end{cases}
$$

We will show that $v^{\prime} \in \operatorname{Hom}\left(\mathbf{L}_{\Sigma^{\mathrm{PP}}}(P), \mathfrak{M}^{\circ}\right)$.

1. $v^{\prime}(\circ \varphi)=\circ v^{\prime}(\varphi)$

If (i) $\sim \circ \varphi \in T$ then by $r_{3}, \circ \circ \varphi \in T$ (so $v^{\prime}(\circ \varphi)=\hat{0}$ ). Thus $v^{\prime}(\circ \varphi)=\hat{0}=\circ\left(v^{\prime}(\varphi)\right.$ ).

If (ii) $\circ \varphi, \varphi \in T$, then by $r_{3}, \circ \circ \varphi \in T$ (so $v^{\prime}(\circ \varphi)=\hat{1}$ ). So $v^{\prime}(\circ \varphi)=\hat{1}=\circ\left(v^{\prime}(\varphi)\right.$ ).

If (iii) $\circ \varphi, \sim \varphi \in T$, so by $r_{3}, \circ \circ \varphi \in T$ (so $v^{\prime}(\circ \varphi)=\hat{1}$ ). So $v^{\prime}(\circ \varphi)=\hat{1}=\circ\left(v^{\prime}(\varphi)\right.$ ).

2. $v^{\prime}(\sim \varphi)=\sim v^{\prime}(\varphi)$

If (i) $\sim \circ \sim \varphi \in T$, then by $r_{3}$ and $r_{7}, o \sim \varphi \notin T$. Then, by $r_{4}, \circ \varphi \notin T$. Thus, by $\mathrm{r}_{3}$ and $\mathrm{r}_{6}, \sim \circ \varphi \in T$ (so $v^{\prime}(\varphi)=v(\varphi)$ ). So $v^{\prime}(\sim \varphi)=v(\sim \varphi)=\sim_{\mathfrak{M}}(v(\varphi))=$ $\sim\left(v^{\prime}(\varphi)\right)$.

If (ii) $\circ \sim \varphi, \sim \varphi \in T$, by $r_{5}, \circ \varphi \in T$ (so $\left.v^{\prime}(\varphi)=\hat{0}\right)$ ). Then $v^{\prime}(\sim \varphi)=\hat{1}=$ $\sim\left(v^{\prime}(\varphi)\right)$.

If (iii) $\circ \sim \varphi, \sim \sim \varphi \in T$, then by $r_{5}, \circ \varphi \in T$ (since $\varphi \in T$, so $v^{\prime}(\varphi)=\hat{1}$ ). Therefore $v^{\prime}(\circ \varphi)=\hat{0}=\sim\left(v^{\prime}(\varphi)\right)$. 
3. $v^{\prime}(\varphi \wedge \psi)=v^{\prime}(\varphi) \wedge v^{\prime}(\psi)$

If (i) $\sim \circ(\varphi \wedge \psi) \in T$, then by $r_{3}$ and $r_{7}$, we have that $\circ(\varphi \wedge \psi) \notin T$. By $r_{12}$, we have that (a) $\circ \varphi, \circ \psi \notin T$, (b) $\circ \varphi \in T$ and $\circ \psi \notin T$ or (c) $\circ \varphi \notin T$ and $\circ \psi \in T$.

(a) If $\circ \varphi, \circ \psi \notin T$ then, by $\mathrm{r}_{3}$ and $\mathrm{r}_{6}, \sim \circ \varphi, \sim \circ \psi \in T$ (so $v^{\prime}(\varphi)=v(\varphi)$ and $\left.v^{\prime}(\psi)=v(\psi)\right)$. So $v^{\prime}(\varphi \wedge \psi)=v(\varphi \wedge \psi)=v(\varphi) \wedge_{\mathfrak{M}} v(\psi)=v^{\prime}(\varphi) \wedge v^{\prime}(\psi)$.

(b) If $\circ \varphi \in T$ and $\circ \psi \notin T$ then, by $\mathrm{r}_{3}$ and $\mathrm{r}_{6}, \neg \circ \psi \in T$ (so $v^{\prime}(\psi)=v(\psi)$ ). By $\mathbf{r}_{8}, \varphi \in T\left(\right.$ so $\left.v^{\prime}(\varphi)=\hat{1}\right)$. Therefore $v^{\prime}(\varphi \wedge \psi)=v(\psi)=v^{\prime}(\psi)=$ $\hat{1} \wedge v^{\prime}(\psi)=v^{\prime}(\varphi) \wedge v^{\prime}(\psi)$

(c) If $\circ \varphi \notin T$ and $\circ \psi \in T$ then the case is analogous to the previous using $r_{9}$. If (ii) $\circ(\varphi \wedge \psi), \varphi \wedge \psi \in T$ then $\varphi, \psi \in T$. By $r_{10}$ and $r_{11}, \circ \varphi, \circ \psi \in T$. (so $\left.v^{\prime}(\varphi)=v^{\prime}(\psi)=\hat{1}\right)$ hence $v^{\prime}(\varphi \wedge \psi)=\hat{1}=v^{\prime}(\varphi) \wedge v^{\prime}(\psi)$.

If (iii) $\circ(\varphi \wedge \psi), \sim(\varphi \wedge \psi) \in T$ then either $\sim \varphi \in T$ or $\sim \psi \in T$. By $r_{13}$, we have that (a) $\circ \varphi, \circ \psi \in T$, (b) $\circ \varphi \in T$ and $\circ \psi \notin T$ or (c) $\circ \varphi \notin T$ and $\circ \psi \in T$.

(a) If $\circ \varphi, \circ \psi \in T$ so $v^{\prime}(\varphi)=\hat{0}$ or $v^{\prime}(\psi)=\hat{0}$. So $v^{\prime}(\varphi \wedge \psi)=\hat{0}=v^{\prime}(\varphi) \wedge$ $v^{\prime}(\psi)$

(b) If $\circ \varphi \in T$ and $\circ \psi \notin T$ then, by $\mathrm{r}_{11} \varphi \notin T$. By $\mathrm{r}_{6} \sim \varphi \in T$ (so $v^{\prime}(\varphi)=\hat{0}$ ). So $v^{\prime}(\varphi \wedge \psi)=\hat{0}=v^{\prime}(\varphi) \wedge v^{\prime}(\psi)$

(c) If $\circ \varphi \notin T$ and $\circ \psi \in T$ then the case is analogous to the previous using $r_{10}$. 4. $v^{\prime}(\varphi \vee \psi)=v^{\prime}(\varphi) \vee v^{\prime}(\psi)$

This case is analogous to the case of $\wedge$, using the corresponding $\vee$ rules.

We may extend the recipe given in the previous result to provide Hilbert-style axiomatizations (that is, with only inference rules with singleton succedents) to the class $\mathcal{M}^{\circ}$ when $\mathcal{M}$ is axiomatized by a Hilbert-style calculus. Before showing how, we will define a set of such traditional Hilbert-style inference rules induced by a set of symmetrical rules. In what follows, when $\Phi=\left\{\varphi_{1}, \ldots, \varphi_{n}\right\} \subseteq L_{\Sigma}(P)(n \geq 1)$, let $\bigvee \Phi=\left(\ldots\left(\phi_{1} \vee \phi_{2}\right) \vee \ldots\right) \vee \phi_{n}$. Also, let $\Phi \vee \varphi=\{\phi \vee \varphi \mid \phi \in \Phi\}$.

Definition 4.2. Let $\mathrm{R}$ be a set of symmetrical rules of inferences. Define the set $\mathrm{R}^{\vee}=$

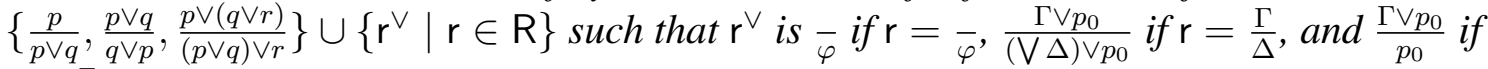
$r=\underline{\Gamma}$, where $p_{0}$ is a propositional variable not occurring in the rules in $R$.

The next result states that, when $R$ is the calculus given by Theorem $4.1, R^{\vee}$ is the Hilbert-style axiomatization we are looking for.

Theorem 4.3. Let $\mathcal{M}$ be a class of $\Sigma^{\mathrm{DM}}$-matrices and let $\mathrm{R}$ be a set of single-succedent rules. If $\vdash_{\mathrm{R}}=\vdash_{\mathcal{M}}=\vdash_{\widehat{\mathcal{M}}}$, then $\vdash_{\left(\mathrm{R} \cup \mathrm{R}_{\mathrm{o}}\right)^{\vee}}=\vdash_{\mathcal{M}^{\circ}}$.

Proof. Analogous to the proof presented in [Marcelino and Rivieccio 2021], but with $\circ$ instead of $\nabla$.

Example 4.4. Let $\mathrm{DM}_{4}$ be the four-element subdirectly irreducible De Morgan algebra as given in [Marcelino and Rivieccio 2021], $\mathfrak{M}_{4}=\left\langle\mathbf{D M}_{4}, \uparrow a\right\rangle$ be the $\Sigma^{\mathrm{DM}}$-matrix that defines the Belnap-Dunn logic $\mathcal{B}$, and let $\mathrm{R}_{\mathcal{B}}$ be the Hilbert calculus used in [Font 1997] to axiomatize $\vdash_{\mathcal{B}}$. Since $\mathcal{B}=\vdash_{\mathfrak{M}_{4}}=\vdash_{\widehat{\mathfrak{M}}_{4}}$ [Marcelino and Rivieccio 2021], we obtain a Hilbert axiomatization for $\mathcal{P} \mathcal{P}_{\leq}=\vdash_{\mathfrak{M}_{4}^{\circ}}=\vdash_{\left\langle\mathbf{P P}_{6}, \uparrow a\right\rangle}$ by adding to $\mathrm{R}_{\mathcal{B}}$ the following rules:

$$
\overline{o \perp} r_{1}^{\vee} \quad \overline{o T} r_{2}^{\vee} \quad \quad \overline{o p p}_{3}^{\vee} \quad \frac{o p \vee r}{o \sim p \vee r} r_{4}^{\vee} \quad \frac{o \sim p \vee r}{o p \vee r} r_{5}^{\vee}
$$




$$
\begin{aligned}
& \frac{o p \vee r}{(p \vee \sim p) \vee r} r_{6}^{\vee} \quad \frac{o p \vee r, p \vee r, \sim p \vee r}{r} r_{7}^{\vee} \quad \frac{o p \vee r}{(\circ(p \wedge q) \vee p) \vee r} r_{8}^{\vee} \\
& \frac{\circ q \vee r}{(\circ(p \wedge q) \vee q) \vee r} r_{9}^{\vee} \quad \frac{\circ(p \wedge q) \vee r, q \vee r}{\circ p \vee r} \mathrm{r}_{10} \quad \frac{\circ(p \wedge q) \vee r, p \vee r}{\circ q \vee r} \mathrm{r}_{11}^{\vee} \\
& \frac{\circ p \vee r, \circ q \vee r}{\circ(p \wedge q) \vee r} r_{12}^{\vee} \quad \frac{\circ(p \wedge q) \vee r}{(\circ p \vee \circ q) \vee r} r_{13}^{\vee} \quad \frac{o p \vee r, \circ q \vee r}{\circ(p \vee q) \vee r} r_{14} \quad \frac{\circ(p \vee q) \vee r}{(\circ p \vee \circ q) \vee r} r_{15}^{\vee} \\
& \frac{o p \vee r, p \vee r}{\circ(p \vee q) \vee r} r_{16}^{\vee} \quad \frac{o q \vee r, q \vee r}{\circ(p \vee q) \vee r} r_{17} \quad \frac{\circ(p \vee q) \vee r}{(\circ p \vee q) \vee r} r_{18} \quad \frac{\circ(p \vee q) \vee r}{(\circ q \vee p) \vee r} r_{19}
\end{aligned}
$$

\section{Conclusions and Future Work}

The logic $\mathcal{I} \mathcal{S}_{\leq}$of involutive Stone algebras, well presented and studied in [Cantú and Figallo 2018], makes use of the operator $\nabla$, which, despite its useful algebraic behaviour, lacks a clear logical and philosophical motivation. By exploring the fact that a perfect operator $\circ$ can be defined in those logics, we investigated an equivalent presentation of the involutive Stone algebras in terms of the so-called perfect paradefinite algebras, highlighting the character of its logic as being a $\mathbf{C}$-system and a D-system. We have also presented Hilbert-style deductive systems (in SET-FMLA and in SET-SET) to extensions of the logics of De Morgan algebras with a perfect operator. Another perspective, which has not been considered in the present paper, comes from [Marcelino and Rivieccio 2021], in which the lattice of super-Belnap logics is shown to be embeddable in the lattice of extensions of $\mathcal{I} \mathcal{S}_{\leq}$. We envisage using the connection between $\mathcal{I} \mathcal{S}_{\leq}$and $\mathcal{P} \mathcal{P}_{\leq}$, as we have shown, to study the extensions of $\mathcal{P} \mathcal{P}_{\leq}$.

\section{References}

Barrio, E. A. and Carnielli, W. (2019). Volume I: Recovery operators in logics of formal inconsistency. Logic Journal of the IGPL, 28(5):615-623.

Belnap, N. D. (1977). A Useful Four-Valued Logic, pages 5-37. Springer Netherlands, Dordrecht.

Caleiro, C. and Marcelino, S. (2019). Analytic calculi for monadic PNmatrices. In Iemhoff, R., Moortgat, M., and Queiroz, R., editors, Logic, Language, Information and Computation (WoLLIC 2019), volume 11541 of LNCS, pages 84-98. Springer.

Cantú, L. M. (2019). Sobre la lógica que preserva grados de verdad asociada a las álgebras de stone involutivas. Master's thesis, Universidad Nacional del Sur, Bahía Blanca, Argentina.

Cantú, L. M. and Figallo, M. (2018). On the logic that preserves degrees of truth associated to involutive Stone algebras. Logic Journal of the IGPL, 28(5):1000-1020.

Font, J. (1997). Belnap's four-valued logic and De Morgan lattices. Logic Journal of the IGPL, 5:1-29.

Marcelino, S. and Rivieccio, U. (2021). Logics of involutive stone algebras. arXiv preprint arXiv:2102.05455.

Marcos, J. (2005a). Logics of Formal Inconsistency. PhD thesis, Unicamp.

Marcos, J. (2005b). Nearly every normal modal logic is paranormal. Logique et Analyse, 48:279-300.

Shoesmith, D. J. and Smiley, T. J. (1978). Multiple-Conclusion Logic. Cambridge University Press. 